\title{
QUASI-REFLEXIVITY AND DUAL NORMS ${ }^{1}$
}

\author{
EMILE BOYD ROTH
}

A Banach space $X$ with norm $p$ will be denoted by $(X, p)$ when it is important to emphasize the norm $p$. The dual norm $p^{*}$ is defined on $X^{*}$ by

$$
p^{*}(f)=\sup \{|f(x)|: p(x) \leqq 1\} .
$$

If $r$ is another norm on $X$ equivalent to $p$, the duals $(X, p)^{*}$ and $(X, r)^{*}$ coincide and will both be denoted by $X^{*}$, and $p^{*}$ and $r^{*}$ are equivalent norms on $X^{*}$. Williams has shown [5] that $(X, p)$ is reflexive if and only if every norm on $X^{*}$ equivalent to $p^{*}$ is the dual of some norm on $X$ equivalent to $p$. The following theorem is a generalization of that result.

Theorem. A Banach space $(X, p)$ is quasi-reflexive of order $\leqq n$, where $n$ is a nonnegative integer, if and only if $X^{*}$ has a subspace $S$ of codimension $\leqq n$ such that for every norm $q$ on $X^{*}$ equivalent to $p^{*}$, there exists a norm $t$ on $X$ equivalent to $p$ such that $t^{*}|S=q| S$.

Proof. (i) Suppose $X$ is quasi-reflexive of order $\leqq n$. By [1, p. 908] there exist a Banach space $(Y, r)$ (quasi-reflexive of the same order as $X)$ and a linear homeomorphism $\phi$ from $(X, p)$ onto $\left(Y^{*}, r^{*}\right)$. Let $J$ be the canonical map from $Y$ into $Y^{* *}$. Let $S=\phi^{*}[J Y]$.

The codimension of $S$ in $X^{*}$ is equal to the codimension of $J Y$ in $Y^{* *}$, which is $\leqq n$. Suppose $q$ is a norm on $X^{*}$ equivalent to $p^{*}$. Define $q_{2}$ on $Y^{* *}$ by $q_{2}(F)=q\left(\phi^{*} F\right)$, where $\phi^{*}$ is the transpose of $\phi$. Then $q_{2}$ and $r^{* *}$ are equivalent norms on $Y^{* *}$ since $q$ and $p^{*}$ are equivalent norms on $X^{*}$ and since $\phi^{*}$ is a linear homeomorphism from $\left(Y^{* *}, r^{* *}\right)$ onto $\left(X^{*}, p^{*}\right)$. Define $q_{3}$ on $Y$ by $q_{3}(y)=q_{2}(J y)$. Note that $r(y)$ $=r^{* *}(J y)$. Now $q_{3}$ and $r$ are equivalent norms on $Y$ since $q_{2}$ and $r^{* *}$ are equivalent norms on $Y^{* *}$ and since $J$ is a linear isomorphism from $Y$ into $Y^{* *}$. Thus $q_{3}^{*}$ and $r^{*}$ are equivalent norms on $Y^{*}$. Define $t$ on $X$ by $t(x)=q_{3}^{*}(\phi x)$. Then $t$ and $p$ are equivalent norms on $X$ since $\phi$ is a linear homeomorphism from $(X, p)$ onto $\left(Y^{*}, r^{*}\right)$. Finally, $t^{*} \mid S$ $=q \mid S$ since for all $y \in Y$ we have

Presented to the Society, January 24, 1969; received by the editors November $15,1968$.

1 This is a portion of the author's doctoral dissertation at The Florida State University under the direction of Professor Ralph D. McWilliams. 


$$
\begin{aligned}
t^{*}\left(\phi^{*} J y\right) & =\sup \left\{\left|\left(\phi^{*} J y\right) x\right|: t(x) \leqq 1\right\}=\sup \left\{|(J y)(\phi x)|: q_{3}^{*}(\phi x) \leqq 1\right\} \\
& =q_{3}^{* *}(J y)=q_{3}(y)=q_{2}(J y)=q\left(\phi^{*} J y\right) .
\end{aligned}
$$

(ii) Suppose that $X^{*}$ has a subspace $S$ of codimension $\leqq n$ such that for every norm $q$ on $X^{*}$ equivalent to $p^{*}$, there exists a norm $t$ on $X$ equivalent to $p$ such that $t^{*}|S=q| S$. Let $J$ be the canonical map from $X$ into $X^{* *}$. We show that $X^{* *}=J X+S^{\perp}$, from which it follows that $X$ is quasi-reflexive of order $\leqq n$ since $\operatorname{dim} S^{\perp} \leqq \operatorname{codim} S \leqq n$ [4, Theorem 3, p. 39]. Let $F \in X^{* *}$. Let $U=\left\{f \in X^{*}: p^{*}(f) \leqq 1\right\}$. For each positive integer $j$, let $V_{j}=\left\{f \in X^{*}:|F(f) \leqq 1 / j|\right\} \cap U$. Each set $V_{j}$ is a balanced, convex, $p^{*}$-bounded, $p^{*}$-closed, $p^{*}$-neighborhood of zero. Consequently $V_{j}$ has a gauge $q_{j}$ which is equivalent to $p^{*}[4$, p. 58]. Thus $V_{j}$ is $q_{j}$-closed, and $V_{j}=\left\{f \in X^{*}: q_{j}(f) \leqq 1\right\}$. By hypothesis there exists a norm $t_{j}$ on $X$ equivalent to $p$ such that $t_{j}^{*}\left|S=q_{j}\right| S$. Let $U_{j}=\left\{f \in X^{*}: t_{j}^{*}(f) \leqq 1\right\}$. Note that the $w\left(X^{*}, X\right)$-topology on $X^{*}$ is independent of the various norms under consideration. Each set $U_{j}$ is $w\left(X^{*}, X\right)$-closed by the Banach-Alaoglu Theorem [4, Theorem 1 , p. 239]. We have $V_{j} \cap S=U_{j} \cap S$ since $q_{j}\left|S=t_{j}^{*}\right| S$. Therefore $V_{j} \cap S$ is relatively $w\left(X^{*}, X\right)$-closed in $S$. Now $F^{-1}(0) \cap U \cap S=\bigcap_{j=1}^{\infty}\left(V_{j} \cap S\right)$, so $F^{-1}(0) \cap U \cap S$ is relatively $w\left(X^{*}, X\right)$-closed in $S$. Let $K$ be the $w\left(X^{*}, X\right)$-closure in $X^{*}$ of $F^{-1}(0) \cap U \cap S$, and let $L$ be the linear span of $K$. Now $K \supseteq p^{*}-\operatorname{cl}\left(F^{-1}(0) \cap U \cap S\right)$ since the $w\left(X^{*}, X\right)$ topology is weaker than the $p^{*}$ topology. Also $p^{*}-\operatorname{cl}\left(F^{-1}(0) \cap U \cap S\right)$ $=U \cap p^{*}-\operatorname{cl}\left(F^{-1}(0) \cap S\right)$ since $F^{-1}(0) \cap S$ is a subspace and since $U$ is the $p^{*}$-unit ball. This $L \supseteq p^{*}-\operatorname{cl}\left(F^{-1}(0) \cap S\right)$. Since $F^{-1}(0) \cap S$ has finite codimension in $X^{*}$, the subspace $L$ is the direct sum of $p^{*}-\operatorname{cl}\left(F^{-1}(0) \cap S\right)$ and some finite-dimensional subspace, and hence $L$ is $p^{*}$-closed by [3, p. 59]. The set $K$ is convex since it is the closure of a convex set [2, Theorem 1, p. 413]. It now follows by a corollary of the Krein-Šmulian Theorem [2, Corollary 9, p. 429] that $L$ is $w\left(X^{*}, X\right)$-closed. We have $L \cap S \supseteq F^{-1}(0) \cap S$ since $L \supseteq p^{*}-\operatorname{cl}\left(F^{-1}(0) \cap S\right)$. Since $K$ is convex and balanced, $L=\operatorname{span} K=\{a k: k \in K$ and $a>0\}$. Thus

$$
L \cap S \subseteq \operatorname{span}(K \cap S)=\operatorname{span}\left(F^{-1}(0) \cap U \cap S\right)=F^{-1}(0) \cap S .
$$

We now have $F^{-1}(0) \cap S=L \cap S$, which is relatively $w\left(X^{*}, X\right)$-closed. Thus $F \mid S$ is $w(S, X)$-continuous by [4, Theorem 3, p. 186].

By [3, Theorem 14.1, pp. 117-118], there exists a $w\left(X^{*}, X\right)$ continuous linear functional $G$ on $X^{*}$ such that $G|S=F| S$. Now $F=G+(F-G) \in J X+S^{\perp}$, and we have $X^{* *}=J X+S^{\perp}$, which completes the proof. 


\section{REFERENCES}

1. P. Civin and B. Yood, Quasi-reflexive spaces, Proc. Amer. Math. Soc. 8 (1957), 906-911

2. N. Dunford and J. T. Schwartz, Linear operators. Part I, Interscience, New York, 1958.

3. J. L. Kelley and I. Namioka, et. al., Linear topological spaces, University Series in Higher Math., Van Nostrand, Princeton, N. J., 1963.

4. A. Wilansky, Functional analysis, Blaisdell, New York, 1964.

5. J. P. Williams, $A$ "metric" characterization of reflexivity, Proc. Amer. Math. Soc. 18 (1967), 163-165.

The Florida State University 